\title{
Materials Informatics
}

\section{John R. Rodgers and David Cebon, Guest Editors}

\begin{abstract}
Global markets are pressuring the materials industries to reduce the time span between materials research and materials development. In particular, current approaches to the development and insertion (deployment) of advanced materials in military systems are too time-intensive and expensive. Despite the large investments in defense systems, insertion is not certain, since materials development is often not linked to the system designer needs, with the results frequently being that the materials do not meet their requirements. There is potentially a high payoff for developing methodologies that will accelerate the insertion of materials, thereby saving millions of investment dollars. All industries engaged in developing advanced materials are searching for new methodologies to solve these problems.

Once a new material has been developed, it must be characterized and modeled. Otherwise, components using it cannot be analyzed, and consequently, it cannot be used in engineering products. Therefore, exploiting the full benefits of new (and existing) engineering materials requires specialized informatics tools for data capture, management, analysis, and dissemination. Advances in computing power, coupled with computational modeling and simulation and materials properties databases, will enable materials scientists and engineers to meet these challenges.

The articles in this issue of MRS Bulletin show a variety of applications of the methodologies that are employed in using materials informatics approaches to materials and engineering product development.
\end{abstract}

Keywords: combinatorial materials science, informatics, performance, properties, simulation, structure, theory.

The term "informatics" has been defined as the use of computer software to gather, store, manipulate, retrieve, and classify information. This issue of MRS Bulletin will focus on materials informatics-the application of computational methodologies to processing and interpreting scientific and engineering data concerning materials. Modern materials science and engineering research produces a large amount of heterogeneous data. Computational methods are then used to organize, manage, interpret, analyze, and visualize these data. Using a combination of computational methods, like density functional theory (DFT), to calculate structure and properties, combined with data mining of properties databases to identify trends and systematics in property data, it is possible, then, to identify suitable candidate starting materials for a variety of materials science and engineering applications. These computational methods are now becoming important tools for use by materials scientists and engineers in materials and product development.
Materials informatics can be broadly divided into three main parts: data generation (synthesis), data management, and knowledge discovery (analysis). New experimental methods, such as combinatorial materials science ${ }^{1}$ and diffusion multiple approaches, ${ }^{2}$ are synthesizing large amounts of structural and property data-physical, chemical, and engineering. Combinatorial materials science is an experimental approach to quickly analyze materials and to test for their physical and structural properties. A diffusion multiple approach is the result of an assembly of three or more metals, in close contact, that is subject to high temperatures to allow thermal interdiffusion. The large amount of data generated by these methods needs to be stored, analyzed, and mined for further applications.

More recently, "computational" combinatorial methods or high-throughput $a b$ initio computations, based on density functional theory (DFT) ${ }^{3}$ (see also the September 2006 issue of MRS Bulletin) are being employed to calculate the structure and properties of materials systematically across a wide parameter space. ${ }^{4}$ DFT, based on quantum mechanical methods, is used in computational materials science for the calculation of structural, electronic, optical, and magnetic properties of materials. These advances, which have been made possible with increases in computational speed, and the parallel development of robust $a b$ initio techniques, are also generating enormous amounts of property data.

The use of these complementary methods-computational experiment and theory-to generate data, coupled with methods for data checking and estimation $^{5}$ for the filling in of "holes" in materials property space, ${ }^{6}$ are providing routes to predict new feasible materials compositions and their properties, ${ }^{7-9}$ thus improving materials design and selection capabilities.

For the successful design of components for engineering applications, data, and knowledge relating to the selection of the material, the component geometry and the processing route need to be optimized to maximize performance and minimize cost and adverse environmental impact. ${ }^{10}$ Other strategies for successful design consist of a systems approach that integrates processing-structure-propertiesperformance (PSPP) relationships where there is a need to link processing parameters of a material to its structure, properties, and performance. ${ }^{11}$ From PSPP relationships, it is possible to establish links between properties and structure, and between structure and processing. Being able to extract such relationships from vast arrays of physical and engineering properties databases and materials processing data facilitates the materials design process.

In this century, materials science will become an ever more important factor in many technologies. The constant quest for new materials with better properties, improved performance of existing materials, decreased materials development costs, and reduced environmental impact ensure the continual and steadily growing importance of systematic materials development and selection.

The Edisonian approach (i.e., trial and error) has been widely used in designing materials. Relying on intuition and experiment, this approach can screen only as many materials for which it is practical to perform experiments. As an illustration, consider Table I, which compares the approximate number of experimentally known materials compositions with the maximum possible number. Most elements and binaries have been studied, but the number of experiments falls drastically 
Table I: Distribution of Chemical Systems.

\begin{tabular}{lccc}
\hline Systems & Experimentally Known & Percent Known & Maximum Number \\
Elements & 100 & $100 \%$ & 100 \\
Binaries & 4,000 & $81 \%$ & 4,950 \\
Ternaries & 8,000 & $5 \%$ & 161,700 \\
Quaternaries & 1,000 & $<1 \%$ & $3,921,225$ \\
\hline
\end{tabular}

for ternaries (three elements), while the properties of quaternaries (four elements) and higher systems are almost unknown. The number of materials studied by this Edisonian process is usually tens, or possibly hundreds, as compared with the virtually infinite number of possible candidates representing tertiary, quaternary, or even more complex compounds, about whose properties we currently know nothing at all, that can be studied using combinatorial methods.

Materials informatics approaches, then, provide an effective way to interpret and use materials properties data in the selection and design of materials and products. The articles in this issue of the MRS Bulletin cover many of the methodologies that have been developed for this purpose.

Morgan et al. ${ }^{12}$ introduce an essential item in the materials development process: crystal structure prediction. Crystal structures, which provide links between the components of the PSPP chain, are important in understanding many materials properties that are symmetry-dependent, ${ }^{13}$ from mechanical to electronic. They show that the inability to predict the crystal structures of a crystalline solid from a knowledge of the chemical composition, alluded to by Maddox ${ }^{14}$ as "one of the continuing scandals in the physical sciences," can be successfully solved using a combination of data mining and $a b$ initio quantum mechanics methods. Predictions using these combined approaches result in $\sim 90 \%$ accuracies if the five most likely structures at each chemical composition are used.

Bligaard et al. cover the applications of electronic structure calculations in the design of metal alloy catalysts. Applying a combination of computational and datamining approaches, they show how the concept of a Pareto-optimal ${ }^{15}$ set can be used to define alloys with favorable catalytic properties, by simultaneously optimizing a multiproperty data set. A Pareto-optimal set produces an infinite set of solutions from which a desired solution can be chosen.

Le Page, who has made significant contributions in the application of data mining and computational approaches to a number of topics-from the crystal chemistry of minerals ${ }^{16}$ to structure types ${ }^{17}$ and physical property calculations ${ }^{18}$ using quantum mechanical calculations-describes a number of applications of the integration of crystal structure databases and computational methods for property data mining. This contribution provides an overview of informatics approaches that can be used in materials development at the crystal structure stage.

Wu describes a computational materials design platform to support materials design across adjacent length scales. This platform, an integration of a variety of computational methods and data-mining techniques, has been used to solve design problems.

Combinatorial materials science $(\mathrm{CMS})^{1}$ aims at discovering new materials using a combination of high-speed chemical synthesis, high-throughput screening, fast data-storing, data screening, and information processing. Takeuchi et al. describe this integration of experimentation and the related data-mining tools as applied to the prediction of new materials. They cover the underlying techniques for data analysis schemes for identifying materials compositions, structure, and properties.

Cebon and Ashby describe in detail the needs for materials data and related software in engineering product development. They systematically cover a number of topics, from data types and data evaluation for the engineering materials selection process to enterprise-wide materials data management. They show how quantitative engineering property data can be integrated with descriptive supporting information in the systematic optimal selection of materials. They also show the need for comprehensive data coverage to facilitate the selection process and discuss important topics such as the correlation between materials properties and the estimation of properties. ${ }^{6,19}$ The application of these last two methods not only improves the quality of the database of materials properties but also makes it possible to include missing data to populate property space. The authors have also described the need for software systems that can automate the key data management tasks: data capture, analysis, deployment, and maintenance. Specific requirements of such systems are flexibility of data management, change control, version control, quality control, and traceability of information.

Arnold focuses on identifying what constitutes "sufficient" data content (i.e., quality and quantity) for developing, characterizing, and validating constitutive models for use in structural analysisparticularly finite element modeling. Constitutive theory concerns the mathematical modeling of the physical response (output) of a material to a given stimulus (input); that input can be a generalized force or displacement. He focuses on the materials information needs of sophisticated nonlinear time- and history-dependent (hereditary) constitutive models. Such models are needed for the accurate prediction of the performance and life of components under arduous operating conditions-for example, cyclic loading at high temperatures. He also describes the informatics infrastructure required for handling the potentially massive amounts of materials data needed to generate these models. Arnold points out that although data for simple materials properties (e.g., density, Young's modulus, ultimate tensile strength, yield stress, coefficient of thermal expansion, etc.) are widely available in the literature, often the corresponding response curves-needed for complex modeling-are not available. Furthermore, the detailed pedigree information that is needed to assess the applicability and quality of the information is often not stored or reported with it. Consequently, much existing materials property data cannot be used for generating the complex constitutive models needed for engineering analysis and design. To address these issues, a paradigm shift is needed in the way that engineering organizations collect, store, manage, and disseminate materials information.

In the aerospace industry, ${ }^{20}$ the introduction of a new class of materials, including the time to develop design practices that fully exploit the performance of the materials, can take 20 years or more. Such development practices are now too long for aerospace materials industries to maintain their competitiveness. Similar issues exist in other materials industries. As a result, the competitiveness and success of materials development depends increasingly on the ability to utilize information, which is abundantly available, to think strategically, faster, and better than the competition and produce new products.

These challenges carry with them the cost of implementation. New technologies to manage information, on its own, do not give a competitive advantage, since information today is widely available and 
technology alone cannot convert data into knowledge. There is thus a need for knowledge creation, and this involves data generation, gathering, evaluation, and organization, and the conversion of data/information into patterns, trends, systematics, and regularities. This can be achieved by domain experts who use the science of the domain coupled with the materials informatics concepts described in this issue in knowledge creation. The return on investment ${ }^{20}$ in using materials informatics approaches in materials research and development makes its inclusion a key resource in product development.

\section{References}

1. X.-D. Xiang, X. Sun, G. Briceno, Y. Lou, K.A. Wang, H. Chang, W.G. Wallace-Freedman, S.-W. Chen, and P. Schultz, Science 268 (1995) p. 1738. 2. J.-C. Zhao, Prog. Mater. Sci. 51 (2005) p. 557.
3. W. Kohn, Rev. Mod. Phys. 71 (1999) p. 1253. 4. D. Morgan, G. Ceder, and S. Curtarolo, Meas. Sci. Technol. 16 (2005) p. 296.

5. M.F. Ashby, Philos. Mag. 85 (2005) p. 11.

6. M.F. Ashby, Proc. R. Soc. London, Ser. A 454 (1998) p. 1301.

7. G. Ceder, Y.-M. Chiang, D.R. Sadoway, M.K Aydinol, Y.-I. Jang, and B. Huang, Nature 392 (1998) p. 694.

8. F. Besenbacher, I. Chorkendoff, B.S. Clausen, B. Hammer, A.M. Molenbroek, J.K. Nørskov, and I. Stensgaard, Science 279 (1998) p. 1913.

9. L.R. Zhao, Q. Yang, K. Chen, D.Y. Seo, J.R. Rodgers, and P.C. Patnaik, in NATO Report RTO-MP-AVT-109, "The Control and Reduction of Wear in Military Platforms" (2004).

10. D. Cebon and M. Ashby, Adv. Mater. Processes 161 (6) (2003) p. 51.

11. G.B. Olson, Science 277 (1997) p. 1237.

12. D. Morgan, J. Rodgers and G. Ceder, J. Phys.: Condens. Matter 15 (2003) p. 4361.

13. J. Nye, Phys. Prop. Cryst. (Oxford University Press, 1985).
14. J. Maddox, Nature 335 (1988) p. 201

15. V. Pareto, Manuali de economia politica (Societa Editrice Libraria, Milano, Italy, 1906).

16. P.H.J. Mercier, P.S. Whitfield, L.D. Mitchell,

I.J. Davidson, Y. Le Page, and T.J. White, Acta Crystallogr. B61 (2005) p. 635.

17. P.H.J. Mercier, L.D. Mitchell, T.J. White, and P.S. Whitfield, Science (2006) submitted.

18. Y. Le Page and J.R. Rodgers, Phys. Chem. Miner. 32 (2005) p. 564.

19. D. Bassetti, Y. Brechet, and M.F. Ashby, Proc. R. Soc. London, Ser. A 454 (1998) p. 1323.

20. R. Schafrik, Technology Transition in Aerospace Industry, Workshop on Accelerating Technology Transition (National Research Council, Washington DC, November 24, 2003)

21. S. Warde, D. Cebon, W. Marsden, P. Coulter, and A. Fairfull, The Materials Information Challenge in Engineering Enterprises (Granta Design Ltd., Cambridge, UK, 2006).

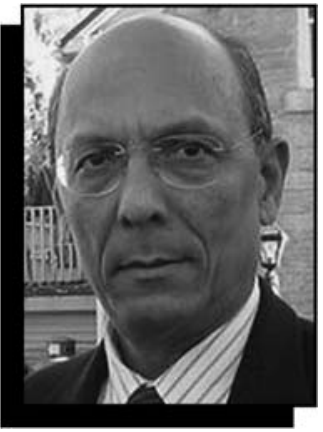

John R. Rodgers

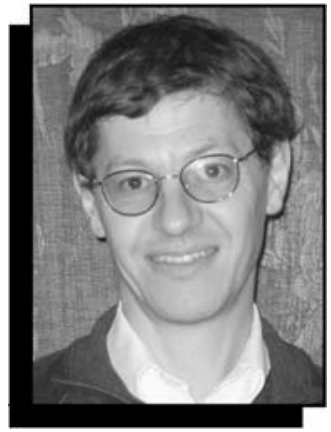

David Cebon

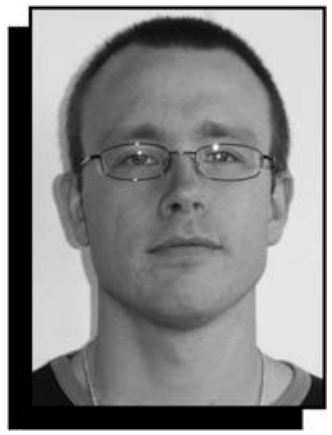

Martin P. Andersson

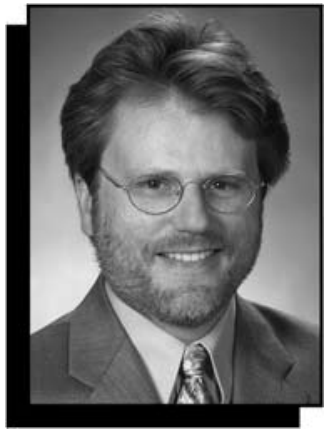

Steven M. Arnold

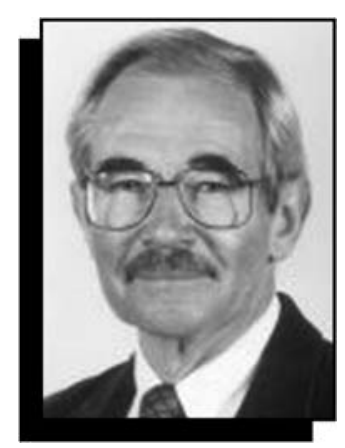

Michael F. Ashby
John R. Rodgers, Guest Editor for this issue of MRS Bulletin, is chief technical officer for Innovative Materials Technologies Inc., a Canadian company specializing in the design of materials, particularly nanocoatings, for gas turbine compressor components and other critical components in the hot section of these engines. He founded Toth Information Systems Inc., a company devoted to the commercial development of inorganic and intermetallic crystal structure databases and computational and visualizing tools for property calculation and data-mining of the physical properties of materials. Prior to founding Toth, Rodgers spent 15 years at the National Research Council of Canada (NRC). Before joining NRC, Rodgers worked at the University of Cambridge. He holds a $\mathrm{PhD}$ degree in crystallography from the University of London.

Rodgers is very involved in the international crystallographic and materials scientific informatics communities.

Rodgers can be reached at Innovative Materials Technologies Inc., 12-B Charles-Bagot St., Gatineau, Quebec J8X 4E1 Canada; tel. 613-9989076, fax 613-990-7444, and e-mail jrodgers@ innovativematerials.com.

\section{David Cebon,}

Guest Editor for this issue of MRS Bulletin, is a professor of mechanical engineering in the Engineering Department at Cambridge University. He holds a bachelor of engineering degree from the University of Melbourne, Australia, and a PhD degree from the University of Cambridge. Cebon is a fellow of the Royal Academy of Engineering. He leads an active research group concerned with the design and dynamics of heavy commercial vehicles, damage mechanisms of pavement materials, and the effects of vehicles on the response and damage of roads and bridges. Cebon is a co-founder of Granta Design Ltd. and has 20 years' experience in software systems for materials information management. He has written numerous papers on the dynamics of heavy vehicles, weighin-motion, road materials, road damage, and materials selection.

Cebon can be reached at Cambridge University, Department of Engineering, Trumpington Street, Cambridge CB2 1PZ, UK; tel. 44-1223-332665, fax 44-1223-332662, and e-mail dc@eng.cam.ac.uk.

Martin P. Andersson is a postdoctoral research associate at the Center for Atomic-Scale Materials Design (CAMD) at the Technical University of Denmark. He received his $\mathrm{PhD}$ degree in 2004 from the University of Lund in Sweden. His earlier research included the field of surface vibrational spectroscopy, where he performed infrared reflection-absorption measurements and developed theoretical models for advanced spectral interpretation, including anharmonic effects from first principles. He is currently focusing on the theoretical treatment of catalytic processes depending on $\mathrm{CO}$ activation.

Andersson can be reached at CAMD, Department of Physics, B. 307, Technical University of Denmark, DK2800 Lyngby, Denmark; tel. 45-4525-3204, fax 454593-2399, and e-mail 


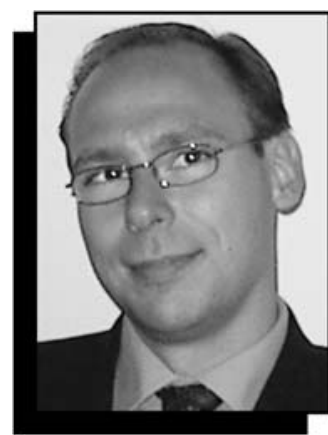

Thomas Bligaard

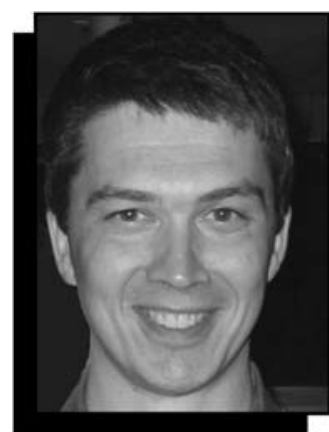

Gerbrand Ceder

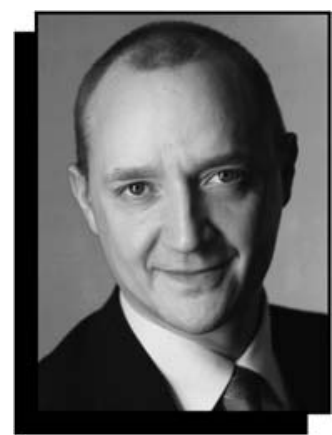

Claus H. Christensen

Ashby can be reached and Lifing Branch, Materials and Structures Division, NASA Glenn Research Center at Lewis Field, 21000

Brookpark Rd., MS 49-7, Cleveland, $\mathrm{OH} 44135$; tel. 216-433-3334, fax 216-433-8300, and e-mail Steven.M.Arnold@ nasa.gov.

Michael F. Ashby is Royal Society Research Professor Emeritus in the Department of Engineering at the University of Cambridge and is the Visiting Professor of Design at the Royal College of Art in London. Prior to Cambridge, he was a professor at Harvard University and held academic positions in Germany and France. He also is a co-founder of Granta Design Ltd.

Ashby is a recipient of numerous awards and honors, including fellow of the Royal Society and member of the American Academy of Engineering. He is the author or co-author of the textbooks Engineering Materials I $\mathcal{E}$ II and Materials Selection in Mechanical Design and of more than 200 papers on mechanisms of plasticity and fracture, powder compaction, mechanisms of wear, methodologies for materials selection, and the modeling of material shaping processes, among other topics. at Cambridge University, Department of Engineering, Trumpington Street, Cambridge CB2 1PZ, UK; tel. 44-1223332635, fax 44-1223332662, and e-mail mfa2@eng.cam.ac.uk.

Thomas Bligaard is an assistant professor at the Center for Atomic-Scale Materials Design (CAMD) at the Technical University of Denmark. He received his PhD degree in 2003 from the Technical University of Denmark, after which he held an EU mobility network research position at the Science Institute, University of Iceland. His research focuses on optimization methods, electronic structure calculations of metals and metal surfaces, and the development of microkinetic modeling tools for the description of surface processes.

Bligaard can be reached at CAMD, Department of Physics, B. 307, Technical University of Denmark, DK2800 Lyngby, Denmark; tel. 45-4525-3209, fax 454593-2399, and e-mail bligaard@fysik.dtu.dk.

Gerbrand Ceder is the R.P. Simmons Professor of Materials Science and Engineering at the

Massachusetts Institute of Technology. He holds

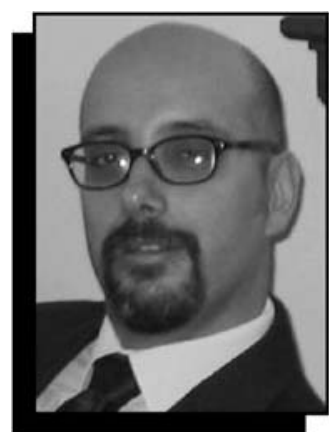

Stefano Curtarolo

an engineering degree from the University of Leuven, Belgium, and a $\mathrm{PhD}$ degree in materials science from the University of California at Berkeley. His research interests lie in firstprinciples predictions of materials properties and structure with a focus on materials for energy generation and storage. Ceder has received the Battery Research Award from the Electrochemical Society, the Career Award from the

National Science Foundation, and the Robert Lansing Hardy Award from TMS. He has published about 200 technical papers.

Ceder can be reached at the Department of Materials Science and Engineering, Massachusetts Institute of Technology, Room 13-5056, 77 Massachusetts Ave., Cambridge, MA 02139 USA; tel. 617-265-5879 and e-mail gceder@ mit.edu.

\section{Claus H. Christensen} (born Claus J.H. Jacobsen) is a professor of chemistry and founder as well as director of the Center for Sustainable and Green Chemistry at the Department of Chemistry at the Technical University of Denmark. He started working at Haldor Topsøe in 1990 and received his MSc degree from the

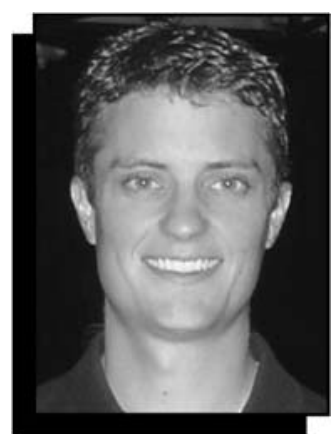

Chris Fischer

University of Copenhagen in 1992. In 2003, he left his position as manager of exploratory research at Haldor Topsøe to join the Technical University of Denmark.

Christensen's research focuses on catalysis, hydrogen storage, and the efficient utilization of bioresources. He is the author of 27 patents and patent applications, and has received the Knud Lind Larsen Prize (2002), Holm's Research Prize (2003), and the Lundbeck Foundation's Prize for Young Scientists (2005).

Christensen can be reached at the Center for Sustainable and Green Chemistry, Department of Chemistry, B. 206, Technical University of Denmark, DK2800 Lyngby, Denmark; tel. 45-4525-2402 and e-mail chc@kemi.dtu.dk.

Stefano Curtarolo is an assistant professor of materials science at Duke University. He received his PhD degree from the Massachusetts Institute of Technology in 2003. Curtarolo's research interests are in alloy theory, surface science, and superconductivity. As a graduate student, he co-developed the technique of datamining of quantum calculations for predicting structure in materials. In 2005, he discovered a 


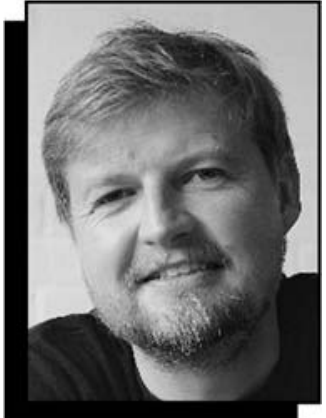

Karsten W. Jacobsen

topological transition in films adsorbed on quasicrystalline surfaces.

Recently, Curtarolo has theoretically developed a lithium monoboride compound that is predicted to have a superconducting temperature comparable to that of magnesium diboride. Together with Gerbrand Ceder and Dane Morgan, he was honored for the best paper of 2005 in the journal CALPHAD.

Curtarolo can be reached at Duke University, Box 90300, 144 Hudson Hall, Research Drive, Durham, NC 27708 USA; tel. 919-6605506, fax 919-660-8963, and e-mail stefano@duke.edu.

\section{Chris Fischer is a $\mathrm{PhD}$} degree candidate in the Materials Science and Engineering Department at the Massachusetts Institute of Technology. He holds a BS degree in metallurgical and materials engineering from the Colorado School of Mines. Fischer's research interests include the application of firstprinciples and machine learning techniques to the prediction of materials properties and materials design.

Fischer can be reached at the Department of Materials Science and Engineering, Massachusetts Institute of Technology, Room 13-5051,

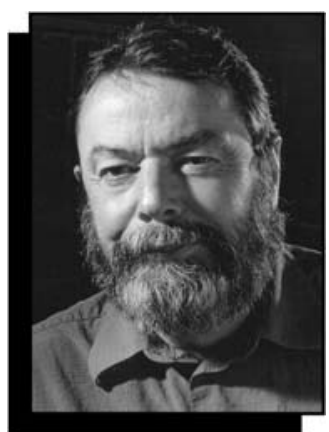

Yvon Le Page

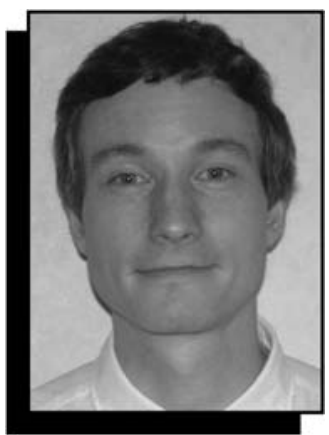

Mikk Lippmaa

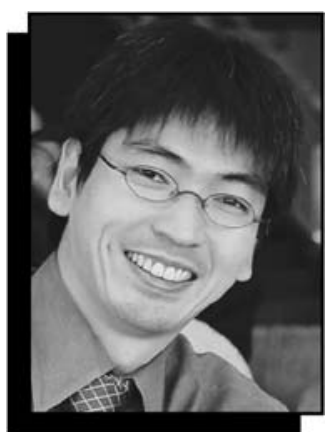

Yuji Matsumoto

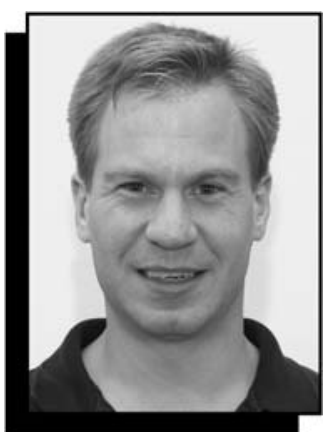

Dane Morgan
77 Massachusetts Ave., Cambridge, MA 02139 USA; tel. 617-253-8127 and e-mail

ccfisch@mit.edu.

Karsten W. Jacobsen is a professor of physics and head of the Department of Physics at the Technical University of Denmark. He received his $\mathrm{PhD}$ degree in 1987 from the University of Copenhagen. He held research appointments at Haldor Topsøe and Cornell University before moving to the Technical University of Denmark in 1988. Jacobsen's research focuses on understanding mechanical and electrical properties of nanostructured materials. He is an elected member of the Danish Academy of Natural Sciences and the Danish Academy of Technical Sciences. Recent honors include the Reinholdt W. Jorck Award for exceptional research in 2004 and Physics Teacher of the Year 2005 at the Technical University of Denmark.

Jacobsen can be reached at CAMD, Department of Physics, B. 307, Technical University of Denmark, DK2800 Lyngby, Denmark; tel. 45-4525-3186, fax 454593-2399, and e-mail kwj@fysik.dtu.dk.

Yvon Le Page is principal research officer at the Institute for Chemical Process and Environmental Technology

(ICPET) of the National Research Council of Canada (NRC). He has worked at NRC since 1977 on research at the crystallographic interface of physics, chemistry, metallurgy, materials science, mineralogy, and biology, both in direct space and in reciprocal space. He is the author or co-author of approximately 175 papers in refereed journals. Le Page has developed novel, symmetrygeneral software that interfaces between crystal structure databases and popular quantum packages, automating the calculation of some physical properties of materials.

Le Page can be reached at the Institute for Chemical Process and Environmental Technology, Room B-9, Bldg. M-12, 1200 Montreal Rd., Ottawa, ON K1A 0R6 Canada; tel. 613-993-2527, fax 613991-2384, and e-mail yvon.le_page@nrc.ca.

Mikk Lippmaa is an associate professor at the University of Tokyo. He received his doctorate degree from the Helsinki University of Technology in 1995. Since then, he has worked at the Tokyo Institute of Technology and the National Institute for Materials Science, developing the pulsed laser ablation technique for combinatorial synthesis of transition-metal oxide thin films. His current work involves the growth of oxide heterostructures for fieldeffect devices.

Lippmaa can be reached at the Institute for Solid State Physics, University of Tokyo, Kashiwanoha 5-1-5, Chiba 277-8581, Japan; tel. 81-4-7136-3315, fax 81-4-7136-3319, and e-mail mlippmaa@ issp.u-tokyo.ac.jp.

Yuji Matsumoto is an associate professor in the materials and structures laboratory at the Tokyo Institute of Technology. He received his BS, MS, and PhD degrees in physical chemistry from the University of Tokyo. Since then, he has devoted his research to surface and interface chemistry and the materials science of $\mathrm{TiO}_{2}$ and related compounds with the aid of combinatorial thin-film technology. His current research interest is flux-mediated epitaxy for single-crystal quality in oxide thin films.

Matsumoto can be reached at the Tokyo Institute of Technology, 4259 Nagatsuta, Midoriku, Yokohama 226-8503
Japan; tel. 81-45-9245314, fax 81-45-924-5377, and e-mail matsumoto@ oxide.msl.titech.ac.jp.

Dane Morgan is an assistant professor in the Department of Materials Science and Engineering at the University of Wisconsin, Madison. He received his PhD degree in physics from the University of California, Berkeley, in 1998. Morgan then worked as a postdoctoral researcher and research associate at the Massachusetts Institute of Technology until joining the faculty at UW-Madison in 2004.

His current research interests focus on ab initio-based thermodynamic and kinetic modeling, with applications to catalysis and catalyst stability in fuel cells, oxide conductors, multicomponent diffusion in irradiated materials, and spin transitions in the Earth's lower mantle.

Morgan has been awarded two Top Paper awards in the area of structure prediction and is a recipient of the $3 \mathrm{M}$ Technical Nontenured Faculty Award.

Morgan can be reached at $244 \mathrm{MSE}$, 1509 University Ave., Madison, WI 53706-1595 USA; tel. 608-265-5879, fax 608-262-8353, and e-mail ddmorgan@ wisc.edu. 


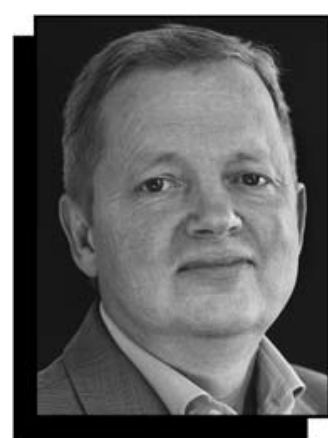

Jens K. Nørskov

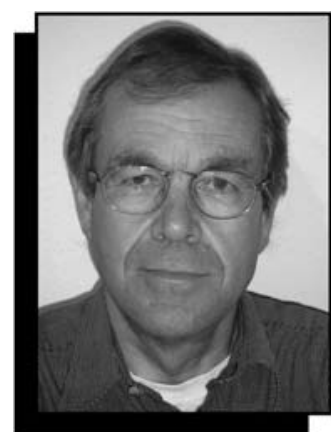

Hans L. Skriver

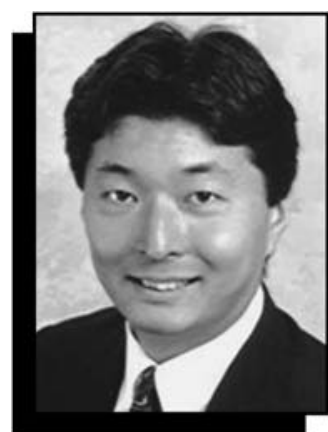

Ichiro Takeuchi

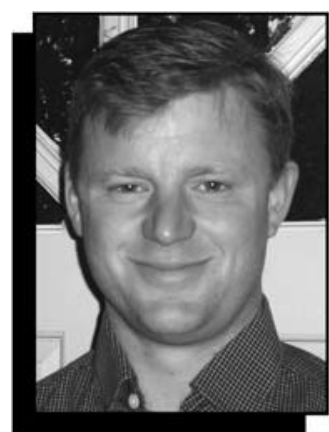

Kevin Tibbetts

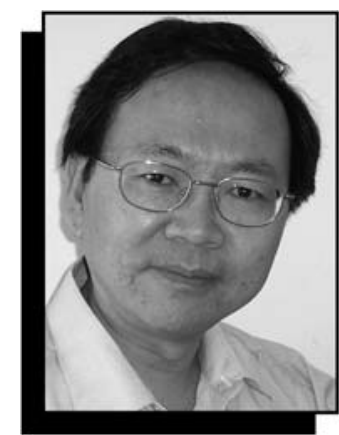

Ping Wu
Department of Physics, B. 307, Technical University of Denmark, DK-2800 Lyngby, Denmark; tel. 45-4525-3175, fax 45-4593-2399, and e-mail norskov@ fysik.dtu.dk.

\section{Hans L. Skriver is a} professor of physics in the Department of Physics at the Technical University of Denmark. $\mathrm{He}$ received his $\mathrm{PhD}$ degree from the Technical University of Denmark in 1973, a DSc degree from the University of Copenhagen in 1985, and was appointed DPhil honoris causa of the University of Uppsala in 2001. Skriver held research positions in Ottawa, Leiden, Stuttgart, the Risø National Lab, and the Nordic Institute for Theoretical Physics before moving to the Technical University of Denmark in 1989.

His research focuses on the development of methodology for describing the electronic structure of alloys.

Skriver can be reached at CAMD, Department of Physics, B. 307, Technical University of Denmark, DK2800 Lyngby, Denmark; tel. 45-4525-3176, fax 454593-2399, and e-mail skriver@fysik.dtu.dk.
Ichiro Takeuchi is an associate professor in the Department of Materials Science and Engineering and the Center for Superconductivity Research at the University of Maryland.

He received his BS degree in physics from the California Institute of Technology in 1987 and his $\mathrm{PhD}$ degree in physics from the University of Maryland in 1996. He was a postdoctoral research fellow at Lawrence Berkeley National Laboratory from 1996 to 1999.

Takeuchi's research interests include novel multilayer thin-film devices, scanning probe microscopes, and the development of combinatorial materials science methodology.

He received the Office of Naval Research Young Investigator Award in 2000 and the National Science Foundation's CAREER Award in 2001.

Takeuchi can be reached at the University of Maryland, Department of Materials Science and Engineering, College Park, MD 20742 USA; tel. 301-4056809, fax 301-405-6327, and e-mail takeuchi@ umd.edu.

Kevin Tibbetts is a third-year PhD student in the Department of Materials Science and Engineering at the Massachusetts Institute of Technology, working in Gerbrand Ceder's group. Tibbetts received his master's degree in engineering in materials science from MIT, studying crystal structure prediction using experimental evidence, and received his BSc degree in engineering physics from the University of Maine. He is investigating inorganic nanotubes using density functional theory. Tibbetts also worked as a data analyst at the Center for Space Research at MIT and was in the U.S. Army for two years.

Tibbets can be reached at 4 Judith Road, Wilmington, MA 01887 USA; tel. 857-2053257 and e-mail kjt@mit.edu.

Ping $\mathbf{W u}$ is a senior scientist in the Institute of High-Performance Computing (IHPC) in Singapore, where he leads a group of more than 30 staff and students conducting computational materials science research. He received his graduate education in Canada in 1992 and postdoctoral training in Australia in 1993. $\mathrm{Wu}$ also held an adjunct professorship in Singapore, including a faculty position with the Singapore-MIT Alliance from 2002 to 2004.

His research focuses on computational materials chemistry, with the goal of designing new chemicals through a combined approach of computation and experiment.

Wu holds a U.S. patent on lead-free solder and has authored more than 70 scientific papers. He has been awarded external research grants from various sources including industry, hospitals, and public research funding agencies totaling more than $\$ 3$ million USD. Also, Wu is a fourtime recipient of the joint Canada-Singapore research grant, and a recipient of the joint China-Singapore research grant. He has completed contract research for many international companies as well as the major hospitals in Singapore.

Wu can be reached at IHPC, Materials and Industrial Chemistry, 1 Science Park Road, \#01-01 The Capricorn, Singapore Science Park II, Singapore 117528; tel. 65-6419-1212, fax 65-6778-0522, and e-mail wuping@ ihpc.a-star.edu.sg. 